\title{
Cost-Utility Analysis of Drug Treatments in Patients with Polypoidal Choroidal Vasculopathy in Thailand
}

\author{
Sermsiri Sangroongruangsri $\mathbb{D D}^{\prime}$ \\ Usa Chaikledkaew ${ }^{1,2}$ \\ Prut Hanusaha ${ }^{3}$ \\ Tanapat Ratanapakorn ${ }^{4}$ \\ Paisan Ruamviboonsuk ${ }^{5}$ \\ 'Social and Administrative Pharmacy \\ Division, Department of Pharmacy, \\ Faculty of Pharmacy, Mahidol University, \\ Bangkok, Thailand; ${ }^{2}$ Mahidol University \\ Health Technology Assessment (MUHTA) \\ Graduate Program, Mahidol University, \\ Bangkok, Thailand; ${ }^{3}$ Department of \\ Ophthalmology, Faculty of Medicine \\ Ramathibodi Hospital, Mahidol \\ University, Bangkok, Thailand; \\ ${ }^{4}$ Department of Ophthalmology, Faculty \\ of Medicine, Khon Kaen University, Khon \\ Kaen, Thailand; ${ }^{5}$ Department of \\ Ophthalmology, Rajavithi Hospital, \\ College of Medicine, Rangsit University, \\ Bangkok, Thailand
}

Purpose: The aim of this study was to estimate the cost-utility and budget impact of pharmacological treatments for the eye with polypoidal choroidal vasculopathy (PCV) in Thailand.

Methods: A Markov model-based cost-utility analysis (CUA) and budget impact analysis were conducted. The lifetime cost and outcomes of PCV treatments were estimated. We discounted costs and outcomes at $3 \%$ per annum. Parameters were estimated using data from published literatures, local cost and utility data, and epidemiology data among Thai patients. Univariate and probabilistic sensitivity analyses were performed to account for parameter uncertainty.

Results: Intravitreal bevacizumab (IVB) resulted in the lowest lifetime cost, followed by IVB plus verteporfin photodynamic therapy (IVB+vPDT) and intravitreal aflibercept (IVA) The combination of IVB or intravitreal ranibizumab (IVR) and verteporfin photodynamic therapy $(I V B+v P D T$ or IVR+vPDT) yielded the highest quality-adjusted life-years (QALY). When compared with IVB from a societal perspective, the incremental cost-effectiveness ratio for patients with PCV receiving IVB+vPDT, IVR+vPDT, IVA were 10,304; 54,135; and 82,738 the United States dollar (USD) per QALY gained, respectively. At the Thai societal willingness to pay threshold of 4884 USD, IVB had the highest probability of being costeffective $(99 \%)$ followed by IVB+vPDT $(1 \%)$. IVB+vPDT could be a cost-effective strategy and required a budget of 12.61 million USD over five fiscal years when the price of verteporfin reduced by approximately $45 \%$.

Conclusion: None of the drug treatments for PCV was cost-effective in the Thai context. The decreased price of verteporfin is required to support the inclusion of IVB+vPDT in the Thai National List of Essential Medicines for the treatment of PCV.

Keywords: PCV, verteporfin, anti-VEGF agent, retinal disease, economic evaluation, Thailand

\section{Introduction}

Polypoidal choroidal vasculopathy (PCV) is a retinal disease that causes severe visual impairment among untreated patients. ${ }^{1}$ Consequently, it leads to diminished visionrelated quality of life (VRQoL) and ability to perform activities of daily living of patients. $^{2} \mathrm{PCV}$ is considered as a subtype of neovascular age-related macular degeneration (nAMD). The prevalence of PCV in Asian patients who were diagnosed with AMD (23.9\% to $54.7 \%$ ) was higher than Caucasian patients (4\% to $9.8 \%)^{3,4}$ A multi-center study conducted in Thailand reported that idiopathic polypoidal choroidal vasculopathy
Correspondence: Usa Chaikledkaew

Tel +662-644-8679 ext 53I7

Fax +662-644-8694

Email usa.chi@mahidol.ac.th 
(IPCV) had the highest prevalent (77.52\%) among Thai patients with choroidal neovascularization (CNV) compared to other retinal diseases. ${ }^{5}$

The main treatments for the PCV management are verteporfin photodynamic therapy (VPDT) and intravitreal injection of anti-vascular endothelial growth factor (antiVEGF) agents, eg, bevacizumab (IVB), ranibizumab (IVR), and aflibercept (IVA). vPDT has played a crucial role in a complete regression of polyps and stabilize or improve visual acuity. ${ }^{1}$ The anti-VEGF drugs can alleviate exudation and hemorrhage to improve or maintain visual acuity (VA). ${ }^{1}$ The short-term effects of IVB and IVR on visual acuity, macular edema, and regression of polypoidal complex in PCV eyes were similar. ${ }^{6}$ Previous studies suggested that VPDT alone or combined with IVR was better than the anti-VEGF monotherapy in a complete polyp regression and reduction in treatment burden and risk of retinal hemorrhage. ${ }^{3,7-11}$

In Thailand, IVB has substituted for vPDT in the National List of Essential Medicines (NLEM) for treating patients with nAMD since 2012. Although both nAMD and PCV patients under the Universal Health Coverage Scheme (UC) can access this treatment with free of charge, they need to pay out of pocket for other PCV treatments when necessary. Due to the high cost of verteporfin and other anti-VEGF drugs, economic evaluation is required to determine the value of money for which intervention should be included into the NLEM. As this study was requested by the Health Economics Working Group (HEWG) under the Subcommittee for the Development of the NLEM, we aimed to conduct cost-utility analysis (CUA) along with budget impact analysis (BIA) of the PCV treatment options in Thailand. The findings will be used to support decision-making on the inclusion of a combination of IVB and vPDT (IVB $+\mathrm{vPDT}$ ) into the Thai pharmaceutical reimbursement list.

\section{Materials and Methods}

\section{Target Population}

The hypothetical cohort comprised Thai patients with unilateral PCV who had never been treated. We set the starting age at 50 years. This assumption was made because most Thai patients with PCV and the patients in the trials $^{9,12,13}$ were elderly aged 50 years old and over. Moreover, more than $80 \%$ of patients were diagnosed with PCV or nAMD in one eye. ${ }^{14,15}$

\section{Model Structure}

The cost-utility analysis (CUA) was performed from societal and payer perspectives. Costs and health outcomes were evaluated over a lifetime horizon. The discount rate of 3\% per annum was applied for both costs and outcomes according to the guideline for health technology assessment in Thailand. ${ }^{16}$

The Markov model with a one-month cycle length (Figure 1) was adapted from the model of Salmon et $\mathrm{al}^{17}$ by adding the transition of patients with hemorrhage to a death health state. It consisted of 8 different health states with arrows indicating possible transitions among them. There were six health states classified according to levels of best-corrected visual acuity (BCVA), ranging from $\leq 25$ Early Treatment Diabetic Retinopathy Study (ETDRS) letters (VA1) to 100 ETDR letters (VA6). We assumed that an equal proportion of the 50-year-old treatmentnaïve unilateral PCV patients entered the Markov model at the health states with BCVA 26-40 (VA2), 41-55 (VA3), and 56-70 (VA4) ETDRS letters. This assumption was based on the eligibility criteria of the EVEREST, ${ }^{11}$ ${\text { EVEREST } \text { II }^{9} \text { and PLANET }}^{18}$ trials. After these patients received the treatments, they would be stable or proceed to the states of having improving vision (gaining 15 letters or more), worsening eyesight (losing 15 letters or more), vitreous hemorrhage (VH) or subretinal hemorrhage (SRH), or death. Patients with VH or SRH might be stable, recover (improve to the VA1 state) or die. Microsoft Excel

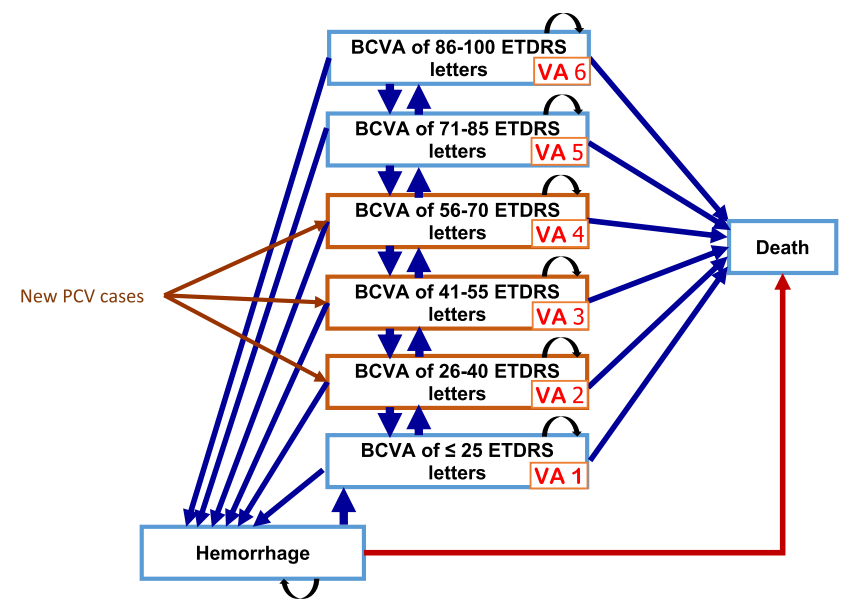

Figure I Schematic diagram of the Markov model representing the clinical progression polypoidal choroidal vasculopathy.

Notes: The arrows show the transition from one health state to another or the same health state in the next cycle.

Abbreviations: BCVA, best-corrected visual acuity; ETDRS, Early Treatment Diabetic Retinopathy Study; VA, level of visual acuity; PCV, polypoidal choroidal vasculopathy. 
was used to construct the Markov model and perform all analyses.

The model assumed that patients in the hemorrhage state had the poorest eyesight (eg, count finger, hand motion, or perception of light) and had no other complications. The PCV treatment was discontinued at this health state. These patients who had massive SRH could receive pneumatic displacement. About $25 \%$ of patients with $\mathrm{VH}$ underwent Par Plana Vitrectomy (PPV), whereas the rest of the patients could recover without any treatment. We assumed that no disease or complication would affect visual acuity and mortality rate in any health state. Additionally, each patient would receive the same medication for treating PCV for a lifetime period.

\section{Interventions and Comparator}

The comparator of this study was IVB monotherapy, as IVB has been a standard of care for patients with nAMD in Thai public hospitals. Currently, it has been listed in the NLEM and has a lower price than other anti-VEGF agents. Although IVB+vPDT was an intervention of the primary interest, other available therapies (ie, IVR monotherapy (0.5 mg/0.05 mL), IVR combined with vPDT (IVR +vPDT), and IVA monotherapy (2 $\mathrm{mg} / 0.05 \mathrm{~mL})$ were also included in the analyses. ${ }^{18,19}$ Our analysis did not include IVA+vPDT because the PLANET trial ${ }^{18,19}$ showed non-inferior results of IVA compared with IVA plus rescue vPDT. Adding vPDT to the IVA would increase only the treatment cost. The treatment patterns during the first two years of IVR and IVR+vPDT were following the EVEREST II trial ${ }^{9,10}$ and we assumed the same treatment frequencies for IVB and IVB+vPDT. We adopted the treatment frequencies of IVA in the years 1 and 2 from the PLANET trial. ${ }^{18,19}$

\section{Model Parameters}

The four main groups of the model input parameters were transition probabilities, costs, utilities, epidemiological data. The parameter values, parameter assumptions, and explanation are presented in Table S1 in Supplementary Materials.

\section{Transition Probabilities and Treatment Efficacy}

The transition probabilities were estimated from proportions of patients with PCV gaining or losing $\geq 15$ ETDRS letters. Currently, there is no randomized controlled trial (RCT) examining the efficacy of the drugs used to treat $\mathrm{PCV}$ in Thailand. We used the year 1 and year 2 results from the EVEREST II trial $^{9}$ (IVR+vPDT arm: $\mathrm{n}=168$, IVR arm: $\mathrm{n}=154)$ and the PLANET trial ${ }^{18,19}($ IVA+sham arm: $n=157$, IVA+active rescue arm: $n=161)$. After year 2 , we used data from LUMINOUS study ${ }^{20}$ along with assumptions. LUMINOUS is an observational study determining safety, effectiveness, and treatment pattern for five years $(n=30,153)$. We could not access to patient-level data of the trials, therefore we assumed that the transitional probability of gaining $\geq 15$ ETDRS letters or the transitional probability of losing $\geq 15$ ETDRS letters was equal across the vision-related health states (VA1 to VA6). Evidence $^{21}$ proved that IVB and IVR had equivalent efficacy in visual improvement among patients with nAMD. Due to the limited efficacy data of IVB in PCV treatment, we assumed that IVB and IVB+vPDT had similar efficacy to IVR and IVR+vPDT, respectively.

The age-specific all-cause mortality rates in the Thai population were derived from the report of Burden of Disease Research Program Thailand (BOD Thailand) in 2013. The PCV condition and related treatments did not affect the probability of death. Regarding ocular serious adverse events (SAE), we included the occurrence of vPDT-related hemorrhage as well as cataracts, retinal detachment, and endophthalmitis due to intravitreal injection of the anti-VEGF drugs. The rates of these SAEs were obtained from the literature. ${ }^{9,18-20}$ These rates were applied for all interventions.

\section{Costs}

Direct medical costs covered costs of medications and administrations, procedures, ocular examination (ie, visual acuity and intraocular pressure measurements, dilation), special investigation (ie, fundus photography, optical coherence tomography (OCT), fundus fluorescein and indocyanine green angiography (FFA and ICGA), and treatment for certain ocular complications. Inputs for resource utilization were obtained from the previous studies. ${ }^{9,18-20}$ The frequencies of drug treatments and clinical visits were assumed to be the same from year 3. Costs of medications, procedures, and ocular examination were derived from the reference price of the Drugs and Medical Supplies Information Centre, the reimbursement price, and primary data from two teaching hospitals in the Central and North-eastern of Thailand. Costs for ocular SAEs Management of any interventions were obtained from literature or calculated from the unit costs of relevant health-care services based on expert opinion. 
Direct non-medical costs (DNMC) included costs of meals, travel, accommodation, house renovation, equipment and accessories required for patients with low vision, productivity loss of caregivers. This cost category was obtained from an unpublished study that collected DNMC from patients with PCV at Ramathibodi hospital. We assumed that DNMC was similar in all health states.

Indirect costs were omitted to avoid double counting in the CUA according to the recommendation from the Thai Health Technology Assessment guidelines. ${ }^{16}$ All costs were converted to the values in 2020 using the consumer price index.

\section{Utilities}

The utility weights for vision-related health states were obtained from the unpublished study. The data were collected from 54 patients with PCV at Ramathibodi hospital using Time Trade-off (TTO) method. The utility values were categorized based on the BCVA of the best-seeing eye. Due to the lack of data from patients with BCVA of 86-100 ETDRS Letters (VA6), we assumed that the utility weight of these patients was equal to those with BCVA of 71-85 ETDRS Letters (VA5). Moreover, the utility weight for patients suffering from hemorrhage complications was assumed to be equivalent to the utility weight for patients with BCVA of $\leq 25$ ETDRS Letters (VA1).

\section{Result Presentation}

We reported total cost in the United States dollar (USD), life-years (LYs), and quality-adjusted life-years (QALYs) of each intervention. Using IVB monotherapy as a reference, the incremental cost-effectiveness ratios (ICER) of each intervention were calculated by dividing an incremental cost by incremental LYs or QALYs. The intervention with the ICER per QALY gained below the cost-effectiveness threshold at 160,000 THB per QALY gained (4884 USD per QALY gained) was considered as a cost-effective option. The total costs and ICER were converted to USD at the rate of 32.76 THB per USD as of 26 August 2021.

\section{Uncertainty Analyses}

\section{Parameter Uncertainty}

Because the main intervention of interest was IVB+vPDT, a one-way sensitivity analysis of this treatment was performed. The discount rates for costs and outcomes varied from $0 \%$ to $6 \%$. While values of the general parameters were varied based on the $95 \%$ confidence interval. The top ten factors influencing the change of ICER were illustrated in a tornado diagram. We employed probabilistic sensitivity analyses (PSA) to examine the uncertainty of all parameters simultaneously for all interventions. The Monte Carlo simulation was run for 1000 iterations. The results of PSA were illustrated by cost-effectiveness acceptability curves (CEAC). The cost-effectiveness plane was developed for IVB+vPDT compared to IVB monotherapy only.

\section{Scenario Analysis}

The clinical data obtained from the EVERST $\mathrm{II}^{9,10}$ and PLANET $^{18,19}$ trials were limited to two years. The potential influence of this short time horizon on the change in ICER value was evaluated.

We performed a threshold analysis. This analysis identifies the medication prices that would result in the PCV treatment being considered cost-effective in the Thai society.

\section{Budget Impact Analysis}

BIA was performed from the payer perspective for five consecutive years. The number of patients with nAMD was obtained from the databases of the National Health Security Office (2012-2019) and the Comptroller General's Department (2019). Meta-analyses were carried out to pool the prevalence and incidence of PCV among Asian patients with nAMD (Table S2 in Supplementary Materials). These parameters were used to estimate prevalent and incident cases of PCV. We assumed that $75 \%$ of the prevalent cases had unilateral PCV. Therefore, the prevalence of the eye receiving the PCV treatment in the first year was 1584 eyes and the incidence of the eye receiving the PCV treatment per year was 1436 eyes. The results from BIA were reported as total annual cost and total cost in five years.

Expert and stakeholder meetings were held to assess the appropriateness of the included interventions, model structure, parameters, and assumptions in accordance with clinical practice in Thailand, as well as preliminary results.

\section{Results}

\section{Base-Case Analysis}

The CUA results are reported in Table 1. The lifetime cost of IVB monotherapy was the lowest, followed by IVB+vPDT, IVA, IVR+vPDT, and IVR, respectively (from 38,216 USD to 112,169 USD). All interventions had the same LY at 18.56 years because the disease and treatments did not increase risk of death. The combination therapy provided the highest 
Table I Results of Cost-Utility Analysis from the Base-Case Analysis

\begin{tabular}{|c|c|c|c|c|c|c|c|c|}
\hline \multirow[t]{2}{*}{ Treatment } & \multirow[t]{2}{*}{$\begin{array}{l}\text { Total Cost } \\
\text { (USD) }\end{array}$} & \multicolumn{2}{|c|}{ Total Effectiveness } & \multirow{2}{*}{$\begin{array}{c}\text { Incremental } \\
\text { Cost } \\
\text { (USD) }\end{array}$} & \multicolumn{2}{|c|}{$\begin{array}{l}\text { Incremental } \\
\text { Effectiveness }\end{array}$} & \multicolumn{2}{|c|}{ ICER } \\
\hline & & LY & QALY & & LY & QALY & $\begin{array}{c}\text { USD per } \\
\text { LY }\end{array}$ & $\begin{array}{c}\text { USD per } \\
\text { QALY }\end{array}$ \\
\hline \multicolumn{9}{|c|}{ Societal perspective } \\
\hline IVB & $\begin{array}{c}38,217 \\
(38,173)\end{array}$ & $\begin{array}{c}|8.56| \\
(I 8.56 \mid)\end{array}$ & $\begin{array}{c}13.902 \\
(\mid 3.889)\end{array}$ & & & & & \\
\hline IVB+vPDT & $\begin{array}{c}47,487 \\
(47,450)\end{array}$ & $\begin{array}{c}|8.56| \\
(|8.56|)\end{array}$ & $\begin{array}{c}14.802 \\
(14.792)\end{array}$ & $\begin{array}{l}927 I \\
(9277)\end{array}$ & $0(0)$ & $\begin{array}{c}0.900 \\
(0.903)\end{array}$ & $\mathrm{N} / \mathrm{A}$ & $\begin{array}{c}10,304 \\
(10,279)\end{array}$ \\
\hline IVR & $\begin{array}{c}112,169 \\
(112,200)\end{array}$ & $\begin{array}{c}|8.56| \\
(I 8.56 \mid)\end{array}$ & $\begin{array}{c}13.902 \\
(13.892)\end{array}$ & $\begin{array}{c}73,953 \\
(74,028)\end{array}$ & $0(0)$ & $\begin{array}{c}0.000 \\
(0.003)\end{array}$ & $\mathrm{N} / \mathrm{A}$ & $\begin{array}{c}\mathrm{N} / \mathrm{A} \\
(28,767,176)\end{array}$ \\
\hline IVA & $\begin{array}{c}70,930 \\
(70,845)\end{array}$ & $\begin{array}{c}|8.56| \\
(I 8.56 \mid)\end{array}$ & $\begin{array}{c}14.298 \\
(14.288)\end{array}$ & $\begin{array}{c}32,714 \\
(32,673)\end{array}$ & $0(0)$ & $\begin{array}{c}0.395 \\
(0.399)\end{array}$ & N/A & $\begin{array}{c}82,738 \\
(81,884)\end{array}$ \\
\hline IVR+vPDT & $\begin{array}{c}86,922 \\
(86,948)\end{array}$ & $\begin{array}{c}|8.56| \\
(|8.56|)\end{array}$ & $\begin{array}{c}\mid 4.802 \\
(\mid 4.793)\end{array}$ & $\begin{array}{c}48,706 \\
(48,775)\end{array}$ & $0(0)$ & $\begin{array}{c}0.900 \\
(0.903)\end{array}$ & N/A & $\begin{array}{c}54,135 \\
(53,994)\end{array}$ \\
\hline \multicolumn{9}{|c|}{ Payer perspective } \\
\hline IVB & $\begin{array}{c}6178 \\
(38,173)\end{array}$ & $\begin{array}{c}|8.56| \\
(|8.56|)\end{array}$ & $\begin{array}{c}13.902 \\
(13.865)\end{array}$ & & & & & \\
\hline IVB+vPDT & $\begin{array}{c}15,449 \\
(47,450)\end{array}$ & $\begin{array}{c}|8.56| \\
(|8.56|)\end{array}$ & $\begin{array}{c}14.802 \\
(14.760)\end{array}$ & $\begin{array}{c}9271 \\
(9261)\end{array}$ & $0(0)$ & $\begin{array}{c}0.900 \\
(0.895)\end{array}$ & N/A & $\begin{array}{c}10,304 \\
(10,348)\end{array}$ \\
\hline IVR & $\begin{array}{c}80,13 \mid \\
(|I| 2,200)\end{array}$ & $\begin{array}{c}|8.56| \\
(|8.56|)\end{array}$ & $\begin{array}{c}13.902 \\
(13.862)\end{array}$ & $\begin{array}{c}73,953 \\
(74,447)\end{array}$ & $0(0)$ & $\begin{array}{c}0.000 \\
(-0.003)\end{array}$ & N/A & $\begin{array}{c}\mathrm{N} / \mathrm{A} \\
\text { (dominated) }\end{array}$ \\
\hline IVA & $\begin{array}{c}38,891 \\
(70,845)\end{array}$ & $\begin{array}{c}|8.56| \\
(|8.56|)\end{array}$ & $\begin{array}{c}\mid 4.298 \\
(\mid 4.270)\end{array}$ & $\begin{array}{c}32,7 \mid 4 \\
(32,7 \mid 8)\end{array}$ & $0(0)$ & $\begin{array}{c}0.395 \\
(0.405)\end{array}$ & N/A & $\begin{array}{c}82,738 \\
(80,688)\end{array}$ \\
\hline IVR+vPDT & $\begin{array}{c}54,884 \\
(86,948)\end{array}$ & $\begin{array}{c}|8.56| \\
(|8.56|)\end{array}$ & $\begin{array}{c}14.802 \\
(14.759)\end{array}$ & $\begin{array}{c}48,706 \\
(48,915)\end{array}$ & $0(0)$ & $\begin{array}{c}0.900 \\
(0.894)\end{array}$ & N/A & $\begin{array}{c}54,135 \\
(54,695)\end{array}$ \\
\hline
\end{tabular}

Notes: Italicized text indicating the perspective of analysis. The values in brackets were results from the probabilistic model.

Abbreviations: IVA, intravitreal aflibercept; IVB, intravitreal bevacizumab; IVB+vPDT, intravitreal bevacizumab in combination with verteporfin photodynamic therapy; IVR, intravitreal ranibizumab; IVR+vPDT, intravitreal ranibizumab in combination with verteporfin photodynamic therapy; ICER, incremental cost-effectiveness ratio; LY, life-year; N/A, not applicable; QALY, quality-adjusted life-year; USD, the United States dollar.

QALY at 13.78 QALY, followed by IVA. From the societal perspective, no intervention was cost-effective in the Thai setting. The ICER of IVB+vPDT, IVR+vPDT, and IVA were 10,304; 54,135; and 82,738 USD per QALY gained, respectively. The results from the payer perspective showed a similar trend, but the total cost of each treatment decreased due to omitting DNMC.

\section{Uncertainty Analyses}

\section{Parameter Uncertainty}

A tornado diagram of IVB + vPDT and IVB (Figure 2) shows the percentage of change in the ICER from the base-case attributable to the change of each parameter. The change in utility weight at a health state with the poorest BCVA level ( 0.256 and 0.804$)$ was the most influential factor $(-35.8 \%$ to $126.2 \%$ change in ICER values). The ICER value was also substantially sensitive to the changes of discounting rates for outcomes and costs, the utility weight at the health state with BCVA 26-40 ETDRS letters, and frequency of vPDT treatment in the combination regimens in year 3 onwards.

The CEAC (Figure 3) showed that IVB had the highest probability of being cost-effective option at $98.95 \%$, followed by IVB+vPDT at $1.05 \%$. Other treatments had no chance to be cost-effective at the Thai societal willingness to pay (WTP) threshold of 4884 USD per QALY gained. The cost-effectiveness plane from 1000 iterations for IVB 


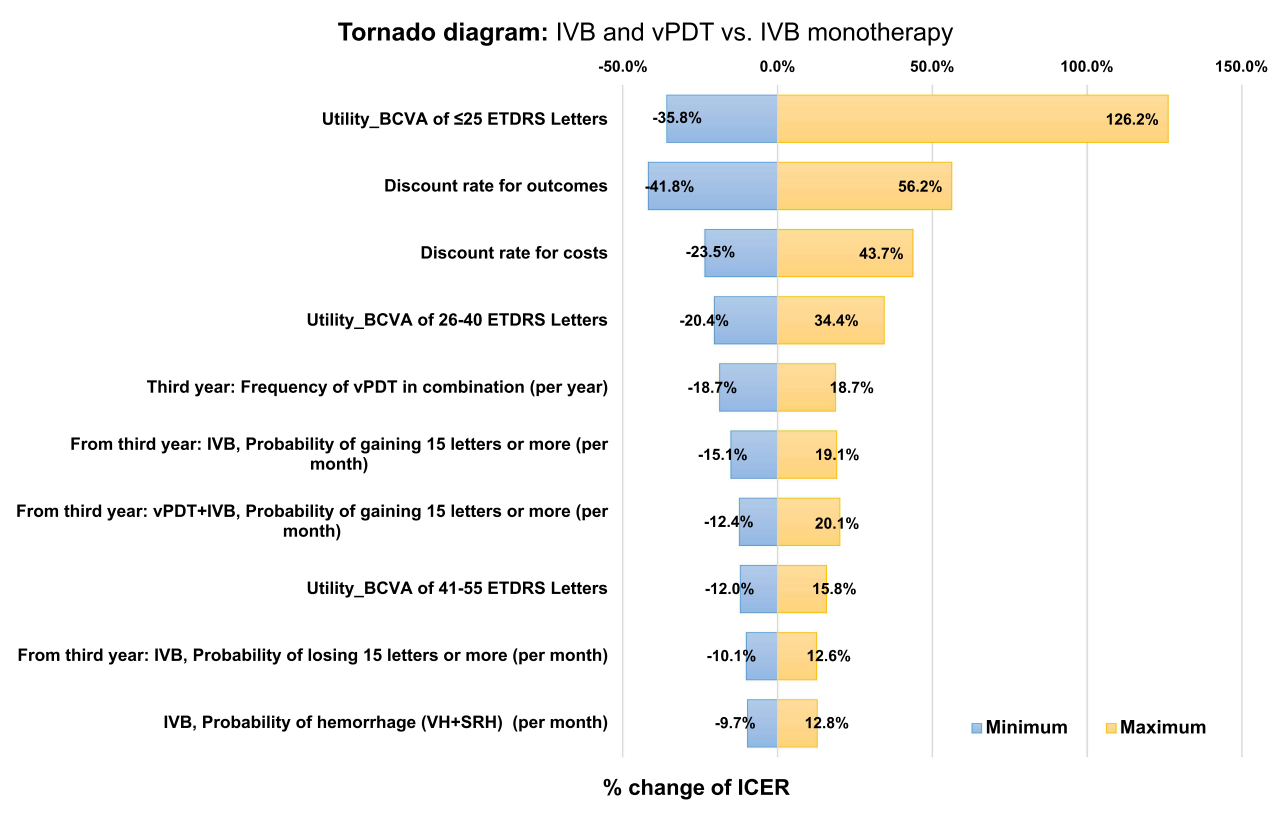

Figure $2 \mathrm{~A}$ tornado diagram of one-way sensitivity analysis for verteporfin combined with intravitreal bevacizumab compared to intravitreal bevacizumab monotherapy. Abbreviations: BCVA, best-corrected visual acuity; ETDRS, Early Treatment Diabetic Retinopathy Study; ICER, incremental cost-effectiveness ratio; IVB, intravitreal bevacizumab; IVB+vPDT, intravitreal bevacizumab in combination with verteporfin photodynamic therapy; SRH, subretinal hemorrhage; VH, vitreous hemorrhage; vPDT, verteporfin photodynamic therapy.

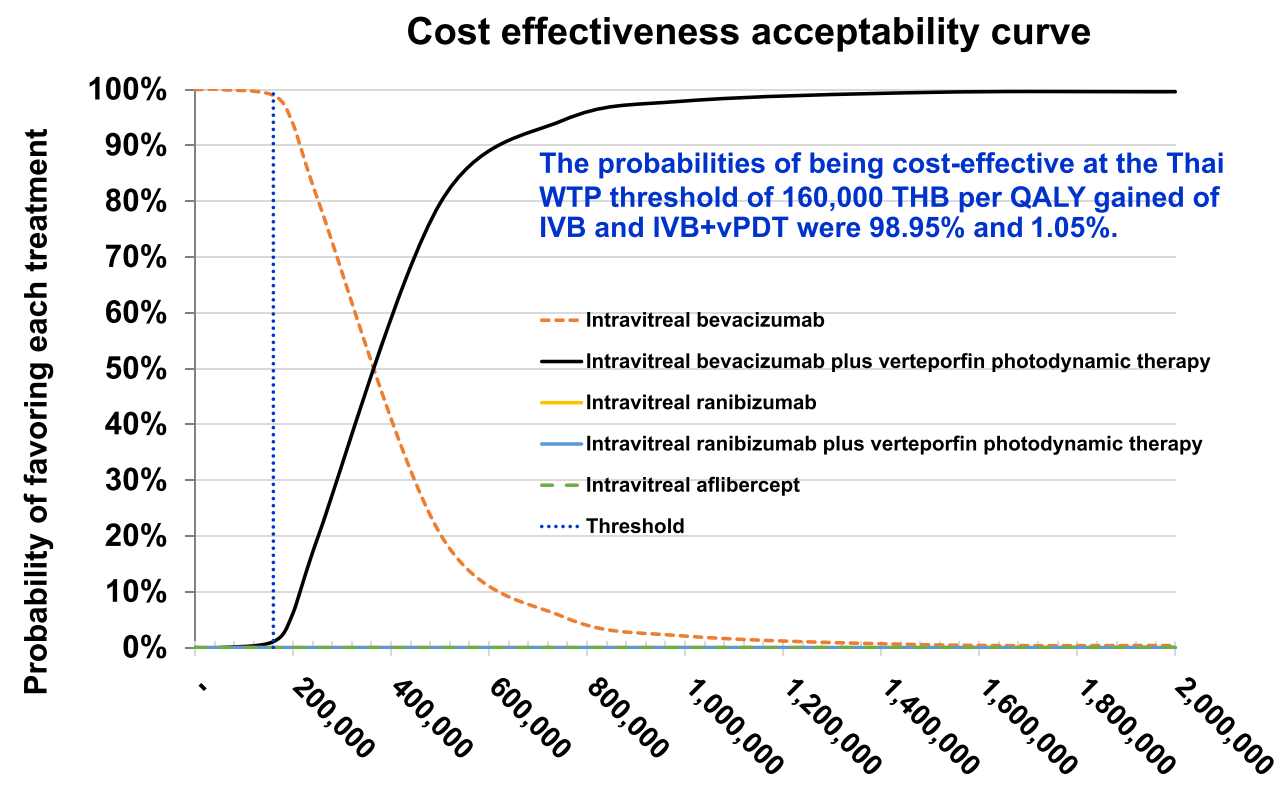

Willingness to pay threshold (THB)

Figure 3 Cost-effectiveness acceptability curves comparing the probabilities of being cost-effective at different willingness-to-pay of the pharmacological treatments for patients with polypoidal choroidal vasculopathy.

Notes: IVR, IVR+vPDT, IVA had zero chance of being cost-effective at all values of ceiling ratio.

Abbreviations: IVB, intravitreal bevacizumab; IVB+vPDT, intravitreal bevacizumab in combination with verteporfin photodynamic therapy; QALY, quality-adjusted life-year; THB, Thai baht; WTP, willingness to pay. 
+vPDT compared with IVB is shown in Figure S1 in Supplementary Materials.

\section{Scenario Analysis}

When analyzing data in the two-year time horizon, IVB + vPDT had least total cost and the lowest ICER value. However, the costs per QALY gained of all interventions in much shorter-time horizon were substantially increased and far beyond the cost-effectiveness threshold (Table 2).

We varied unit cost of the drugs to identify their costs at the Thai societal WTP threshold. This threshold analysis suggested that ICER of IVB+vPDT versus IVB would reach the cost-effectiveness threshold when the cost of verteporfin was 637.64 USD per vial or reduced by $44.7 \%$ from the original price (Figure S2 in Supplementary Materials). IVA monotherapy had a chance of being cost-effective option when the price of aflibercept reduced to 169.34 USD per vial $(85.7 \%$ reduction in price). IVR and IVR + vPDT were not cost-effective at any price of ranibizumab.

\section{Budget Impact Analysis}

At base-case analysis, total budget required for providing IVB and IVB+vPDT treatments was 15 million USD and 40 million USD over five consecutive years (Table S3 in Supplementary Materials). The additional budget required for providing IVB $+v P D T$ instead of IVB was 5 million
USD annually. The proportion of the medication cost was much greater than the monitoring cost (Figure S3 in Supplementary Materials).

If the cost of verteporfin could be reduced to 637.64 USD per vial, the total budget required for IVB+vPDT reduced to 28 million USD for five fiscal year and the incremental budget of using IVB+vPDT instead of IVB was 2.5 million USD per year.

\section{Discussion}

The prevalence of $\mathrm{PCV}$ in nAMD patients is relatively high in Asian populations compared to other populations. ${ }^{3,4}$ The benefits of current PCV treatments, particularly the combination of VPDT and anti-VEGF injections, were proven that could improve both anatomic and visual outcomes and minimize the burden of intravitreal anti-VEGF injections. ${ }^{9-11}$ Despite economic evaluations have played a crucial role in decision-making on the value for money of high-cost medications, there was a few economic evaluations determining the cost-utility of the PCV treatments. ${ }^{17,22,23}$ This study is the first CUA of PCV treatments conducted in Thailand under both societal and payer perspectives over the lifetime horizon. We employed two-year clinical and resources used data from the two landmark RCTs, the EVEREST $\mathrm{II}^{9,10}$ and PLANET $^{18,19}$ studies. The cost and quality of life of patients with PCV were obtained from unpublished studies which

Table 2 The Results Under the Two-Year Time Horizon from a Societal Perspective

\begin{tabular}{|c|c|c|c|c|c|c|c|c|}
\hline \multirow[t]{2}{*}{ Treatment } & \multirow[t]{2}{*}{$\begin{array}{l}\text { Total Cost } \\
\text { (USD) }\end{array}$} & \multicolumn{2}{|c|}{ Total Effectiveness } & \multirow{2}{*}{$\begin{array}{l}\text { Incremental } \\
\text { Cost } \\
\text { (USD) }\end{array}$} & \multicolumn{2}{|c|}{$\begin{array}{l}\text { Incremental } \\
\text { Effectiveness }\end{array}$} & \multicolumn{2}{|c|}{ ICER } \\
\hline & & LY & QALY & & LY & QALY & $\begin{array}{l}\text { USD per } \\
\text { LY }\end{array}$ & $\begin{array}{c}\text { USD per } \\
\text { QALY }\end{array}$ \\
\hline \multicolumn{9}{|c|}{ Societal perspective } \\
\hline IVB & 4896 & 1.958 & 1.493 & & & & & \\
\hline IVB+vPDT & 7210 & 1.958 & 1.515 & 2314 & 0.000 & 0.023 & $N / A$ & 102,754 \\
\hline IVR & 19,186 & 1.958 & 1.493 & 14,290 & 0.000 & 0.000 & $\mathrm{~N} / \mathrm{A}$ & $\mathrm{N} / \mathrm{A}$ \\
\hline IVA & 19,273 & 1.958 & 1.515 & 14,377 & 0.000 & 0.022 & $N / A$ & 644,755 \\
\hline IVR+vPDT & $|6,54|$ & 1.958 & 1.515 & 11,645 & 0.000 & 0.023 & $N / A$ & 517,186 \\
\hline \multicolumn{9}{|c|}{ Payer perspective } \\
\hline IVB & 1378 & 1.958 & 1.493 & & & & & \\
\hline IVB+vPDT & 3691 & 1.958 & 1.515 & 2314 & 0.000 & 0.023 & $\mathrm{~N} / \mathrm{A}$ & 102,754 \\
\hline IVR & 15,667 & 1.958 & 1.493 & 14,290 & 0.000 & 0.000 & $N / A$ & $N / A$ \\
\hline IVA & 15,755 & 1.958 & 1.515 & 14,377 & 0.000 & 0.022 & $\mathrm{~N} / \mathrm{A}$ & 644,755 \\
\hline IVR+vPDT & 13,023 & 1.958 & 1.515 & 11,645 & 0.000 & 0.023 & $N / A$ & 517,186 \\
\hline
\end{tabular}

Note: Italicized text indicating the perspective of analysis.

Abbreviations: IVA, intravitreal aflibercept; IVB, intravitreal bevacizumab; IVB+vPDT, intravitreal bevacizumab in combination with verteporfin photodynamic therapy; IVR, intravitreal ranibizumab; IVR+vPDT, intravitreal ranibizumab in combination with verteporfin photodynamic therapy; ICER, incremental cost-effectiveness ratio; LY, life-year; N/A, not applicable; QALY, quality-adjusted life-year; USD, the United States dollar. 
these data were collected from the local setting to reflect Thai real-life practice.

IVB undoubtedly cost the least for the lifetime of Thai patients with PCV, followed by IVB+vPDT. This finding was in line with a study by Feng et $\mathrm{al}^{23}$ which found that replacing IVB with IVR could reduce cost per QALY by approximately a factor of 5 . The combination therapies resulted in greater health outcomes than the monotherapies. Combining vPDT with the anti-VEGF treatment could reduce the frequency of the anti-VEGF injections and result in a lower lifetime cost. However, the alternative PCV therapies were still not cost-effective in the Thai context. IVB+vPDT and IVA could be cost-effective treatments by reducing prices of verteporfin and aflibercept by approximately $45 \%$ and $86 \%$, respectively. IVB+vPDT was likely to be a promising treatment strategy to be included in the Thai NLEM for PCV because it generated a better health outcome than IVB and had a low budget impact, especially after the price negotiation to reach the price at the cost-effectiveness threshold.

The sensitivity analyses demonstrated the robustness of the ICER in the presence of parameter uncertainty. The results from one-way sensitivity analysis indicated that the ICER for IVB+vPDT compared to IVB was most sensitive to VRQoL. Literature suggested that the most commonly used EQ-5D is not suitable for measuring in patients with eye disease affecting vision. [31] Therefore, we obtained utility data from the study using the TTO method for a better reflect VRQoL of the patients with visual impairment. Similar CUA studies conducted in Singapore ${ }^{22}$ and the United States ${ }^{23}$ employed mathematic equations to estimate the QALY, while one study ${ }^{17}$ employed the utility weights from patients with diabetic macular edema from another study.

Compared to other CUA, ${ }^{22,23}$ a study by Salmon et al ${ }^{17}$ extrapolated data from the EVEREST study which was a small trial with the six-month follow-up period for a lifetime horizon. This study highlighted limited evidence of the incidence of hemorrhagic events which could heavily influence on the CUA's results. Among the previous studies, only the study by Salmon et $\mathrm{al}^{17}$ and our study included the cost of ocular adverse event management that might result from the interventions of interest. Moreover, we perceived that the patients and their families need to bear out-of-pocket expenses due to continuous treatments and follow-up visits as well as their poor visions. Thus, we also considered this cost category in the analysis from a societal perspective.
Recently, the global supply shortage of verteporfin $\left(\right.$ Visudyne $^{\circledR}$ ) has had a drastic impact on this drug access and timely treatment among patients with sight-threatening retina conditions. ${ }^{24}$ This issue would remain problematic worldwide until early $2022 .{ }^{24}$ There is no alternative generic version of verteporfin. As a result, proper management of the remaining stock for the high priority of patients at risk is required. Besides, governments should formulate measures and policies to ensure a sustainable supply of this drug to prevent delayed treatments causing severe vision loss.

This study had some limitations which the result interpretation and policy implementation should be done with caution. Firstly, we could not access the patient-level data of the trials. The assumption of similar vision improvement or worsening across the levels of visual acuity of the patients was made. The treatment effect data derived from the trials were available for up to 2 years. As a result, the long-term treatment effects were extrapolated from the observational study $^{20}$ together with expert opinion. There has been no RCT comparing the efficacy of IVB with other treatments for PCV eyes. Thus, we assumed that the equivalent efficacy between IVB and IVR. Secondly, VRQoL data was obtained from a small number of participants with low vision. As the ICER was sensitive to the change of utility among these groups of patients, further study with a larger sample size might be required to improve the robustness of the CUA's result. Finally, patients in the model would proceed to another health state that was classified by visual acuity levels. Therefore, the benefit of vPDT in a complete polyp regression in a long run might not be captured by this model. Concerning the application of our finding, the opinions from retinal specialists on using a combination of IVB and vPDT and inclusion of such a treatment into the NLEM for PCV treatment are indispensable.

\section{Conclusion}

IVB+vPDT might be a promising alternative to IVB for treating patients with $\mathrm{PCV}$. This treatment could improve both anatomic and vision outcomes as well as reduce the burden of anti-VEGF injections. Although the alternative drugs for PCV management remained not cost-effective, IVB+vPDT and IVA monotherapy could be value for money in Thailand and cause lower budget impact at the reduced prices of verteporfin and aflibercept. Apart from the inclusion of the cost-effective treatment for patients with PCV in the NLEM, the governments and stakeholders should develop 
measures and policies for ensuring the continuous availability of sight-saving medications and enhancing drug access.

\section{Ethics Approval}

This CUA used anonymized and aggregated data related to quality of life and direct non-medical costs from another unpublished study conducted by our research team. The Institutional Review Boards (IRB) in Mahidol University approved this study (COA. No. MURA2018/1030) through the expedited review procedure. All participants were required to provide written informed consent before to be part of the study. All procedures performed in the study were in compliance with international guidelines for human research protection such as Declaration of Helsinki, the Belmont Report.

\section{Acknowledgments}

We are very grateful to Miss Saowalak Turongkaravee and Miss Kankamon Kittrongsiri for technical support on data analysis.

\section{Funding}

This study was supported by the Thai Food and Drug Administration through the Health Economics Working Group (HEWG) under the Subcommittee for the Development of the National List of Essential Medicines (NLEM).This paper represents the views of the authors. The HEWG under the Subcommittee for the Development of the NLEM in Thailand, appointed from 2019 to 2021, reviewed the technical component of the work but is not responsible for the study findings and the dissemination of the findings.

\section{Disclosure}

Dr Sermsiri Sangroongruangsri report grants from the Thai Food and Drug Administration through the Health Economics Working Group (HEWG) under the Subcommittee for the Development of the National List of Essential Medicines (NLEM), during the conduct of the study. The authors report no other conflicts of interest in this work.

\section{References}

1. Cheung CMG, Lai TYY, Ruamviboonsuk P, et al. Polypoidal choroidal vasculopathy. Ophthalmology. 2018;125(5):708-724. doi:10.1016/j. ophtha.2017.11.019

2. Brown MM, Brown GC, Sharma S, Busbee B. Quality of life associated with visual loss: a time tradeoff utility analysis comparison with medical health states. Ophthalmology. 2003;110(6):1076-1081. doi:10.1016/S0161-6420(03)00254-9
3. Koh AH, Chen LJ, Chen SJ, et al. Polypoidal choroidal vasculopathy: evidence-based guidelines for clinical diagnosis and treatment. Retina. 2013;33(4):686-716. doi:10.1097/IAE.0b013e3182852446

4. Lim LS, Mitchell P, Seddon JM, Holz FG, Wong TY. Age-related macular degeneration. Lancet. 2012;379(9827):1728-1738. doi:10.1016/S0140-6736(12)60282-7

5. Bhoomibunchoo C, Yospaiboon Y, Thoongsuwan S, et al. Idiopathic polypoidal choroidal vasculopathy in Thai patients with clinical and angiographic choroidal neovascularization. Clin Ophthalmol. 2017;11:317-322. doi:10.2147/OPTH.S126226

6. Cho HJ, Baek JS, Lee DW, Kim CG, Kim JW. Short-term effectiveness of intravitreal bevacizumab vs. ranibizumab injections for patients with polypoidal choroidal vasculopathy. Korean J Ophthalmol. 2012;26(3):157-162. doi:10.3341/kjo.2012.26.3.157

7. Qian T, Li X, Zhao M, Xu X. Polypoidal choroidal vasculopathy treatment options: a meta-analysis. Eur J Clin Invest. 2018;48(1): e12840. doi:10.1111/eci.12840

8. Han LH, Yuan LF, Liang X, Jia X, Zhang ML. Combined therapy versus anti-vascular endothelial growth factor monotherapy for polypoidal choroidal vasculopathy: a meta-analysis. Int J Ophthalmol. 2017;10(8):1280-1289.

9. Koh A, Lai TY, Takahashi K, et al. Efficacy and safety of ranibizumab with or without verteporfin photodynamic therapy for polypoidal choroidal vasculopathy: a randomized clinical trial. JAMA Ophthalmol. 2017;135(11):1206-1213. doi:10.1001/ jamaophthalmol.2017.4030

10. Lim TH, Lai TYY, Takahashi K, et al. Comparison of ranibizumab with or without verteporfin photodynamic therapy for polypoidal choroidal vasculopathy: the EVEREST II randomized clinical trial. JAMA Ophthalmol. 2020;138(9):935-942. doi:10.1001/ jamaophthalmol.2020.2443

11. Koh A, Lee WK, Chen LJ, et al. EVEREST study: efficacy and safety of verteporfin photodynamic therapy in combination with ranibizumab or alone versus ranibizumab monotherapy in patients with symptomatic macular polypoidal choroidal vasculopathy. Retina. 2012;32(8):1453-1464. doi:10.1097/IAE.0b013e31824f91e8

12. Wong CW, Cheung CM, Mathur R, et al. Three-year results of polypoidal choroidal vasculopathy treated with photodynamic therapy: retrospective study and systematic review. Retina. 2015;35 (8):1577-1593. doi:10.1097/IAE.0000000000000499

13. Wong CW, Wong TY, Cheung CMG. Polypoidal choroidal vasculopathy in Asians. J Clin Med. 2015;4(5):782-821. doi:10.3390/ jcm4050782

14. Sho K, Takahashi K, Yamada H, et al. Polypoidal choroidal vasculopathy: incidence, demographic features, and clinical characteristics. Arch Ophthalmol. 2003;121(10):1392-1396. doi:10.1001/ archopht.121.10.1392

15. Dilokthornsakul P, Chaiyakunapruk N, Ruamviboonsuk P, et al. Health resource utilization and the economic burden of patients with wet age-related macular degeneration in Thailand. Int J Ophthalmol. 2014;7(1):145-151.

16. Working Group on the Guidelines for Health Technology Assessment in Thailand. Guidelines for Health Technology Assessment in Thailand. 1st ed. Nonthaburi, Thailand: The Graphico Systems Co., Ltd.; 2009.

17. Salmon HA, Chalk D, Stein K. How lack of information hampers decision making in ophthalmology. $\mathrm{Br} J$ Health Care Manag. 2017;23(1):28-38. doi:10.12968/bjhc.2017.23.1.28

18. Lee WK, Iida T, Ogura Y, et al. Efficacy and safety of intravitreal aflibercept for polypoidal choroidal vasculopathy in the PLANET study: a randomized clinical trial. JAMA Ophthalmol. 2018;136 (7):786-793. doi:10.1001/jamaophthalmol.2018.1804

19. Wong TY, Ogura Y, Lee WK, et al. Efficacy and safety of intravitreal aflibercept for polypoidal choroidal vasculopathy: two-year results of the aflibercept in polypoidal choroidal vasculopathy study. $A m$ J Ophthalmol. 2019;204:80-89. doi:10.1016/j.ajo.2019.02.027 
20. LUMINOUSTM: study to observe the effectiveness and safety of ranibizumab through individualized patient treatment and associated outcomes. Basel: Novartis Pharma AG; April 12, 2017.

21. Chakravarthy U, Harding SP, Rogers CA, et al. Alternative treatments to inhibit VEGF in age-related choroidal neovascularisation: 2-year findings of the IVAN randomised controlled trial. Lancet. 2013;382(9900):1258-1267. doi:10.1016/S0140-6736(13)61501-9

22. Doble B, Finkelstein EA, Tian Y, et al. Cost-effectiveness of intravitreal ranibizumab with verteporfin photodynamic therapy compared with ranibizumab monotherapy for patients with polypoidal choroidal vasculopathy. JAMA Ophthalmol. 2020;138(3):251-259. doi:10.1001/ jamaophthalmol.2019.5628
23. Feng PW, Lin J, Sakurada Y, et al. Cost and outcomes analysis of polypoidal choroidal vasculopathy (aneurysmal type 1 neovascularization) treatment strategies. J Vitreoretin Dis. 2020;5(3):227-231. doi: $10.1177 / 2474126420946599$

24. The Royal College of Ophthalmologists. Drugs shortage: continuing shortage of visudyne (verteporfin); 2021. Available from: https:// www.rcophth.ac.uk/2021/07/shortage-of-visudyne/. Accessed August $31,2021$.

\section{Publish your work in this journal}

ClinicoEconomics and Outcomes Research is an international, peerreviewed open-access journal focusing on Health Technology Assessment, Pharmacoeconomics and Outcomes Research in the areas of diagnosis, medical devices, and clinical, surgical and pharmacological intervention. The economic impact of health policy and health systems organization also constitute important areas of coverage. The manuscript management system is completely online and includes a very quick and fair peer-review system, which is all easy to use. Visit http://www.dovepress.com/testimonials.php to read real quotes from published authors. 